\title{
CAPACITIVE SENSOR FOR THE MEASUREMENT OF VFTO IN GIS
}

\author{
V. Vinod Kumar and Joy Thomas M.
}

\begin{abstract}
A capacitive sensor based on the theory of electric field measurements using floating electrodes has been developed for the measurement of VFTO and the same has been fitted to the metallic enclosure of a $145 \mathrm{kV}$ GIS experimental test setup available in the laboratory. The sensor has been calibrated insitu with sinusoidal, impulse and step voltage excitations of the HV bus of the $145 \mathrm{kV}$ GIS and the results are presented. The sensor was then used to measure the $50 \mathrm{~Hz}$ restrike pattern as well as the VFTO waveforms during a simulated disconnector switching operation in the 145 $\mathrm{kV}$ GIS test setup. It has been demonstrated that the same sensor is able to faithfully measure both the $50 \mathrm{~Hz}$ restrike pattern as well as the ns duration VFTO waveforms successfully.
\end{abstract}

\section{Introduction}

During disconnector switching operation, a number of restrikes occur across the contacts leading to generation of Very Fast Transient Overvoltage (VFTO) in Gas Insulated Substation (GIS). VFTO are characterised by a very fast rise time of the order of $1 \mathrm{~ns}$ or higher followed by high frequency oscillations in the range of a few $\mathrm{kHz}$ to $\mathrm{MHz}[1]$. The peak value could be as high as 2.5 p.u or higher. As mentioned before, since the VFTO have small rise-times, it is very difficult to practically measure the VFTO waveform in an actual system. Inorder to accomplish this, special wide-band sensor with a bandwidth of few $\mathrm{Hz}$ to atleast few hundreds of $\mathrm{MHz}$ have to be developed.

A capacitive sensor based on the theory of electric field measurements using floating electrodes have been developed. This paper discusses the basic theory, calibration and performance of the sensor developed.

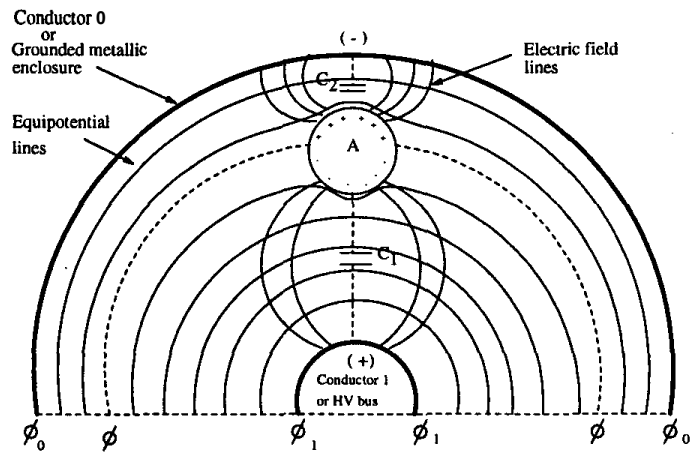

Figure 1: Sketch Illustrating the General Principle of the Electric Field Sensor

\section{Basic theory of the sensor}

The concept of potential measurements with a floating electrode may be illustrated by considering an arbitrary disposition of conductors 0 and 1 as shown in figure 1 where conductors ' 0 ' and ' 1 ' give rise to the field and ' $A$ ' represents the sensing element. The size of ' $\mathbf{A}$ ' of course is grossly exaggerated. The most desirable configuration is that which disturbs the field, the least.

'A' will float to a potential $\phi$ that lies between $\phi_{0}$ and $\phi_{1}$. Since there is no physical connection between ' $A$ ' and either electrode and it is assumed ' $A$ ' does not emit or collect charge from the surrounding medium, then the net charge on ' $A$ ' is zero. As the figure 1 shows, however, there are regions of positive and negative surface charge on ' $A$ ' and in case of the two dimensional geometry shown, these regions are divided by the equipotential surface corresponding to the floating potential of 'A'.

When the floating electrode does not disturb the equipotentials originally present between the two electrodes, it reaches instantaneously a potential corresponding to a fraction of the potential difference existing across the electrode gap corre- 
sponding to the floating potential of ' $A$ '. Its response time theoretically is the time required to redistribute the charges on ' $A$ '.

The capacitance $\mathrm{C}_{2}$ between the region of positive surface charge and the electrode at the potential $\phi_{0}$ may be defined as

$$
C_{2}=\frac{\int \sigma_{+} \cdot d A_{+}}{\phi-\phi_{0}}=\frac{Q_{+}}{\phi-\phi_{0}}
$$

Similarly, the capacitance $\mathrm{C}_{1}$ between the region of negative surface charge and the electrode at potential $\phi_{1}$ is

$$
C_{1}=\frac{\int \sigma_{-} . d A_{-}}{\phi-\phi_{1}}=\frac{Q_{-}}{\phi-\phi_{1}}
$$

Since 'A' is an isolated conductor,

$$
\int \begin{aligned}
& \sigma_{+} \cdot d A_{+} \\
& \sigma_{+} \cdot d A_{+}
\end{aligned} \int \sigma_{-} \cdot d A_{-}=0
$$

Consequently,

$$
C_{2}\left(\phi-\phi_{0}\right)+C_{1}\left(\phi-\phi_{1}\right)=0
$$

The quantity $\phi$ then becomes

$$
\phi=\frac{C_{2} \cdot \phi_{0}+C_{1} \cdot \phi_{1}}{C_{1}+C_{2}}
$$

Since we are interested only in differences in potential, we may arbitrarily

set $\phi_{0}=0$ and obtain

$$
\phi=\frac{C_{1} \cdot \phi_{1}}{C_{1}+C_{2}}
$$

This result, which is very well known from electrostatics, forms the basis of voltage measurements with capacitive dividers.

When $\mathrm{C}_{2} \gg \mathrm{C}_{1}$, the floating electrode is closely coupled to ground. For this case of great practical importance for VFTO measurement in a GIS, it may be noted from equation 6 that

$$
\phi=\frac{C_{1}}{C_{2}} \cdot \phi_{1}
$$

hence,

$$
\mathbf{4 1}=\frac{C_{2}}{C_{1}} \cdot \phi
$$

The measurement of $\phi$ in principle, should be sufficient to determine $\phi_{1}$. There are some practical difficulties, however because the quantity ' $\mathrm{C}_{1}$ ' $\&$ ' $\mathrm{C}_{2}$ ' depends on how the sensor is installed and the field configurations in its vicinity. Consequently, to obtain $\phi_{1}$, calibration of the sensor is required in its environment.

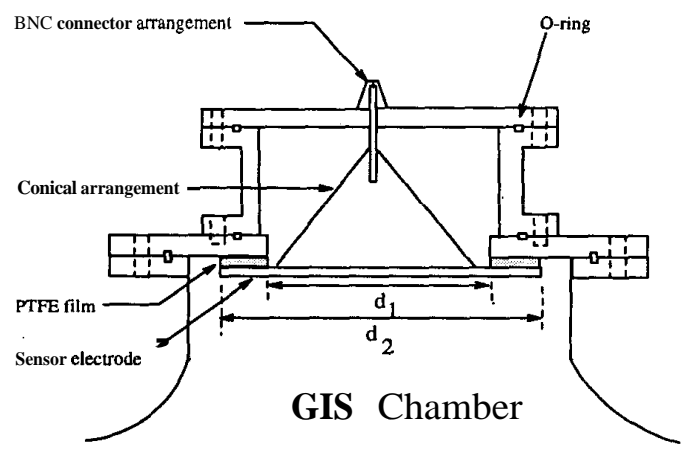

Figure 2: Sketch showing the capacitive sensor

The sensor described in the previous section require for practical purpose, a metering resistor to measure the potential $\phi$ of the conductor ' $A$ '. Depending on the value of this metering resistor, the measured voltage becomes proportional to the voltage on the HV bus or its derivative. If the sensor is terminated with a high impedance measuring resistor $(\gg 1 \mathrm{MHz})$, then the cut-off frequency falls below $50 \mathrm{~Hz}$ and the sensor can be calibrated with $50 \mathrm{~Hz}$ voltages also.

\section{Development of the Sensor}

A capacitive sensor based on the above principle has been developed and tested in a $145 \mathrm{kV}$ GIS test set up. The high voltage capacitor $\left(C_{1}\right)$ of the capacitive divider will be the capacitance formed between the high voltage bus and the sensor electrode. It is to be noted that $\mathrm{SF}_{6}$ is acting as the dielectric in between the high voltage bus bar and the sensor electrode [2][4], $\mathrm{C}_{1}$ for the chosen geometry is found to be $57 \mathrm{pF}$.

The low voltage capacitor is formed between the sensor electrode and the ground. PTFE (Poly Tetra Fluoro Ethylene) film has been used as the dielectric between the sensing electrode and the ground. It's value is found to be $18 \mathrm{nF}$. The dielectric constant of PTFE is found to be constant for a wide range of frequencies and hence is chosen for this application [3]. The output is taken from the low voltage capacitor through a conical termination as shown in figure 2.

\subsection{Insitu calibration of the sensor}

Calibration of the sensor was done with sinusoidal, impulse and step voltages with the capacitive sensor mounted on a $145 \mathrm{kV}$ rated GIS test set-up. A sketch of the GIS setup is shown in figure 3. The sphere gaps are made to touch each other and sinusoidal input from a $150 \mathrm{kV}, 50 \mathrm{kVA}$ transformer was applied to the bushing terminal of the GIS set-up. The input and output wave- 


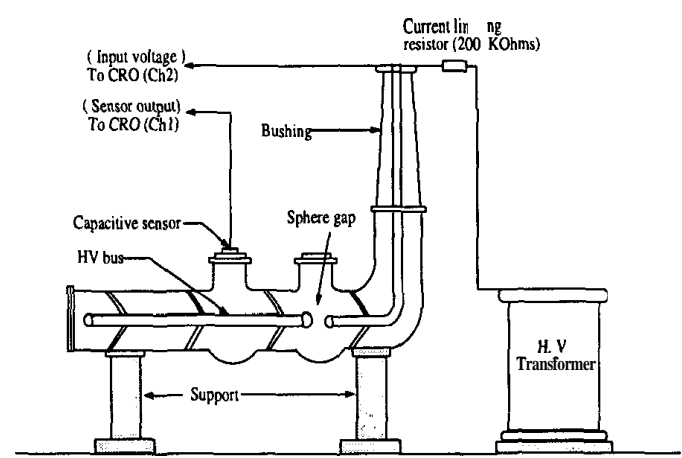

Figure 3: Experimental arrangement of the GIS test setup

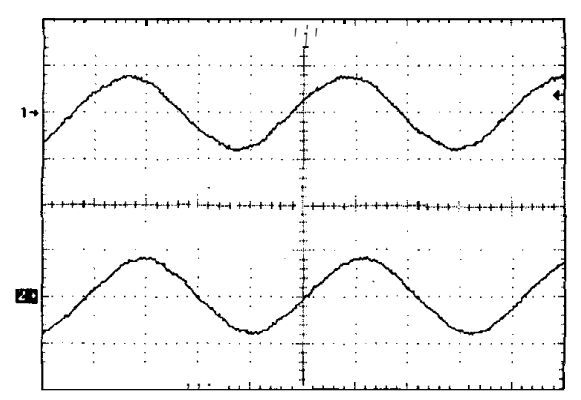

Chl: 2 V (Sensor Output), Ch2: $1.25 \mathrm{kV}$ (HV bus voltage), Time: $5 \mathrm{~ms}$

Figure 4: Oscillograms of sensor output for sinusoidal voltage excitation of the HV bus

forms are measured simultaneously. Various measurements were done at different voltages of $2 \mathrm{kV}$, $2.6 \mathrm{kV}, 5.2 \mathrm{kV}$ and $7.8 \mathrm{kV}$. Figure 4 shows the waveform that is captured from the above experiment with an input voltage of $2 \mathrm{kV}$ (peak-topeak). Channel $\mathbf{1}$ is the sensor output and Channel $\mathbf{2}$ is the input voltage applied to the bushing of the GIS. For a voltage of $2 \mathrm{kV}$ applied to HV bus, the sensor output is 4.4 Volts and hence the capacitive sensor ratio is found to be $\mathbf{4 5 4}$ for $\mathbf{5 0}$ $\mathrm{Hz}$ voltages.

For calibration of the sensor with impulse voltage, a $160 \mathrm{kV}$ impulse voltage generator was used for calibration of the sensor. The same setup as shown in figure 3 was used. The input voltage had a time-to-front of $\mathbf{1 . 3} \mu \mathrm{s}$ and a time-to-tail of $\mathbf{4 8}$ ps. When the above mentioned impulse voltage is applied, the output is found to have a wave-shape having the same time-to-front and time-to-tail as the input voltage as shown in figure 5 . Channel 1 shows the impulse voltage applied to the HV bus and Channel 2 shows the output of the sensor. The capacitive sensor divider ratio is found to be 456.
The step response of the capacitive sensor is carried out by applying a square wave of $4 \mathrm{~ns}$ rise time to the inner HV conductor and measuring the output pulse response of the capacitive sensor. The same experimental setup is as shown in figure 3. The length of the cable was chosen as small as possible so as to minimise its effect on the overall measurement. It is seen that for an input square wave of $\mathbf{4} \mathrm{ns}$ rise-time, the output is found to have a rise time of $6.4 \mathrm{~ns}$ as can be seen from figure 6 . The output of the sensor is found to have oscillations and kinks which are as a result of the reflections from the various components and terminations that the wave sees. However the overall wave-shape of the step input wave is maintained and also the pulse width and the rise time are also found to match. The magnitude of the input pulse is $\mathbf{1 1}$ Volts and the average output magnitude of the sensor is found to be $24 \mathrm{mV}$ for the given cable. Hence the voltage ratio of the sensor is experimentally found to be $\mathbf{4 5 8}$.

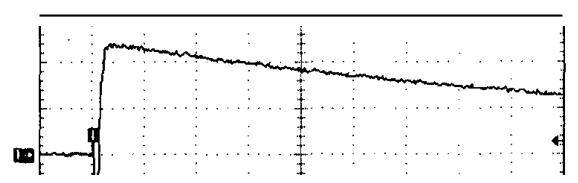

$2 \rightarrow$

Chl: $22.8 \mathrm{kV}$ (HV bus voltage), Ch2: 50V (Sensor Output), Time: $5 \mu \mathrm{s}$

Figure 5: Oscillograms of sensor output for impulse voltage excitation of the HV bus

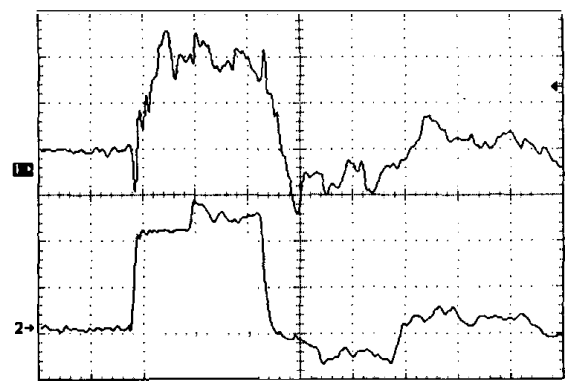

(a) Chl: $15 \mathrm{mV}$ (Sensor Output), Ch2: 5V (Calibration Step Voltage), Time: 20 ns

Figure 6: Step response of the sensor

The insitu calibration of the sensor done with $50 \mathrm{~Hz}$ sinusoidal voltage, impulse voltage and step 
voltage has shown that the divider ratio remains almost constant at $456 \pm 2$ thereby demonstrating that the sensor has a flat response for a wide frequency range.

Inorder to have a constant ratio which is important for measurement of the signal, the same cable is deliberately used for calibration at all frequency ranges.

\section{Generation and Measure- ment of VFTO in an exper- imental test set-up}

The HV bus was excited with a sinusoidal voltage of $50 \mathrm{~Hz}$ from a $150 \mathrm{kV}, 50 \mathrm{kVA}$ testing transformer.

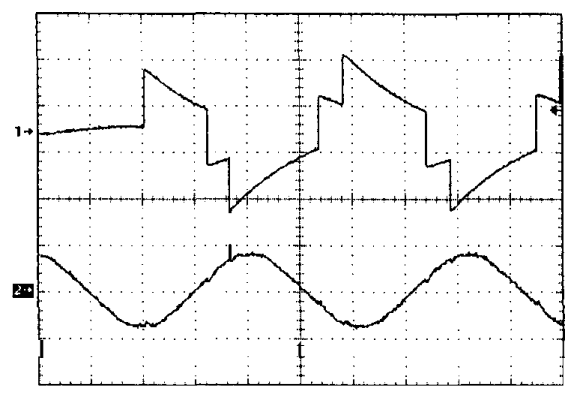

Figure 7: Oscillogram of restrike pattern measured by the sensor with $0.6 \mathrm{~mm}$ gap, Chl: 20V(Sensor output), Ch2: $25 \mathrm{kV}$ (HV bus voltage), Time: $5 \mathrm{~ms}$, Pressure $=3 \mathrm{~atm}$

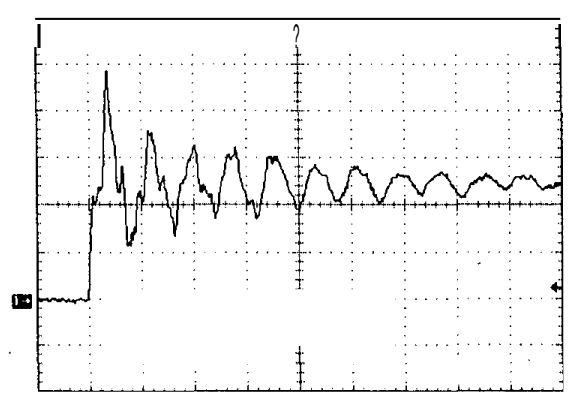

Figure 8: Oscillogram of VFTO measured by the sensor with a gap of $0.6 \mathrm{~mm}, \mathrm{Chl}$ : $10 \mathrm{~V}$ (Sensor output), Time: $50 \mathrm{~ns}$, Pressure $=3 \mathrm{~atm}$

The sphere gap shown in the sketch is used to simulate the disconnector switching operation and thereby the generation of VFTO. Inorder to study the arcing patterns due to restrikes at the sphere gap, measurements were done at $5 \mathrm{~ms}$ time scale, along with the VFTO measurements which were done with $50 \mathrm{~ns}$ time bases. The bus duct was pressurised with $\mathrm{SF}_{6}$ gas at different pressures and the voltage excitation to the HV bus was adjusted using a variac and the recording of both the input and output of the sensor was done when a restrike occurs.

It can be seen from figure 7 that there was always more than a single restrike which ultimately leads to the step shaped waveform or the restrike pattern. Every time there is a restrike, a VFTO is generated as shown in figure 8 and this is separately captured with the $50 \mathrm{~ns}$ time base. The oscillograms amply demonstrate that the sensor developed is able to faithfully measure the $50 \mathrm{~Hz}$ restrike pattern as well as the high frequency fast rise time VFTO waveforms.

\section{Conclusion}

The capacitive sensor developed is a wide band sensor which can measure both the low frequency restrike pattern as well as the ns rise-time VFTO. Also the divider ratio of the sensor is found to be constant at $456 \pm 2$ for a wide range of frequencies.

\section{References}

[1] N. Fujimoto, S. A. Boggs, "Characteristics of GIS Disconnector-Ind uced Short Risetime Transients Incident on Externally Connected Power System Components", IEEE Transactions on Power Delivery, Vol.3, No.3, July 1988, pages 961-970.

[2] H. Okubo, H. Murase, S. Yanabu, "Measurement of Fast Transient Overvoltage and Particle-Initiated Flashover Characteristics in GIS", Second Workshop and Conference on EHV Technology, August 1989, pages 13.

[3] S. A. Boggs, N. Fujimoto, "Techniques and Instrumentation for Measurement of Transients in Gas Insulated Switchgear", IEEE Transactions on Electrical Insulation, VolEI19, No.2, April 1984, pages 87-92.

[4] P. Osmokrovic, D. Petkovic, O. Markovic, "Measuring Probe for Fast Transients Monitoring in Gas Insulated Substations", IEEE Transactions on Instrumentation and Measurement, Vol.46, No.1, February 1997, pages 36-44. 